\title{
Comparative Study of Analytic Solution and Numerical Solution of Baseband Symbol Signal Based on Optimal Generic Function
}

\author{
Mingxin Liu $\mathbb{D}^{1},{ }^{1}$ Wei Xue $\mathbb{D}^{1},{ }^{1}$ Sergey B. Makarov, ${ }^{2}$ Junwei Qi, ${ }^{1,3}$ and Beiming Li $\mathbb{D}^{1}$ \\ ${ }^{1}$ College of Information and Communication Engineering, Harbin Engineering University, Harbin 150001, China \\ ${ }^{2}$ Institute of Physics, Nanotechnology and Telecommunications, St. Petersburg State Polytechnical University, \\ St. Petersburg 195251, Russia \\ ${ }^{3}$ College of Computer Science and Technology, Harbin Engineering University, Harbin 150001, China \\ Correspondence should be addressed to Beiming Li; libeiming@hrbeu.edu.cn
}

Received 12 August 2019; Accepted 21 October 2019; Published 28 November 2019

Academic Editor: Ivan D. Rukhlenko

Copyright (c) 2019 Mingxin Liu et al. This is an open access article distributed under the Creative Commons Attribution License, which permits unrestricted use, distribution, and reproduction in any medium, provided the original work is properly cited.

\begin{abstract}
In this paper, the optimal mathematical generic function model is established using the minimum out-of-band energy radiation criterion. Firstly, the energy limit conditions, boundary constraints, and peak-to-average ratio constraints are applied to the generic function model; thus, the analytical solutions are obtained under different parameters. Secondly, a single symbol signal energy constraint condition and boundary constraint condition are added to the generic function model; thus, the numerical solution of the different parameters is obtained. In the process of solving the analytical solution, the partial solution process is simplified to solve the analytical solution, and there are also digital truncation problems. In addition, the corresponding order of the Lagrange differential equation increases by a multiple of 2 when the parameter $n$ increases, which makes the solution extremely complicated or even impossible to solve. The numerical solution is in line with the current development trend of digital communication, and there is no need to simplify the solution process in the process of solving the numerical solution. When the parameter $n$ and the Fourier series $m$ take different values, the obtained symbol signals can also meet the needs of different communication occasions. The relevant data of the above research process were solved by a MATLAB software simulation, which proves the correctness of the method and the superiority of the numerical method.
\end{abstract}

\section{Introduction}

Frequency resources are becoming increasingly scarce with the rapid development of communication technology, which has resulted in some frequency bands even being reused. To improve the spectrum utilization of the allocated frequency bands, the method of channel space division multiplexing (multibeam antenna, beam-converting and synthesizing antenna, polarized antenna, etc.) is often used to achieve the purpose of reusing the same frequency band. However, there are still issues to be solved, such as the optimization of signal waveforms, modulation and coding methods, and the development of signal forming and processing equipment in band-limited [1-5]. In the communication system, the spectrum characteristics of the bandwidth occupied by the modulated signal and the out-of-band energy radiation mainly depend on the form of the baseband symbol signal
$[6,7]$. The symbol signals used in the past are usually some simple empirical signal waveforms. For example, the application of rectangular waves in signals, such as quadrature phase shift keying modulation (QPSK) and offset quadrature phase shift keying modulation (OQPSK) and sine and cosine waves in the minimum shift keying signal (MSK) baseband modulation, have been used for the purpose of achieving a compressed band by a simple combined signal of the empirical waveform and by a filter [8-12]. The fourth-generation wireless communication uses OFDM as the basic modulation method, and rectangular signals are used as the baseband to transmit signals. The out-of-band radiation is extremely high although the transmission efficiency is improved; therefore, the band utilization still needs further improvement [13-16]. Many scholars have also optimized the design of the OFDM spectrum utilization algorithm. Zhang and Letaief obtained the spectral efficiency of the 
optimal solution by using an adaptive subcarrier bit algorithm to improve spectrum utilization [17]. Alternative modulation schemes for the fifth-generation wireless communications, such as GFDM and FBMC, have both significantly improved the frequency band utilization of subcarriers. However, they also have higher requirements for the choice of baseband signals [18-21].

To improve the spectrum efficiency of the baseband signal symbol of the communication system and improve the reliability of the transmission of the communication system, this paper establishes a mathematical generic function model by introducing minimum out-of-band energy radiation criterion. To establish the corresponding Lagrange equation, the boundary model and the energy limit of the symbol signal are imposed on the function model. A quadrature modulated baseband symbol signal with spectral fast rolling-off characteristics is obtained starting from the analytical method and the numerical method. According to the solution process of the two solution methods, the calculation method and the calculation amount are compared. The analytical solution significantly simplifies the partial solution process in the solution process. The obtained symbol signal is an approximate solution of the optimal symbol signal to be obtained by initially establishing the constraint added in the generic function model. In addition, high-quality symbol signals suitable for different communication occasions can be obtained by the application of the Fourier series in the numerical solution process under the constraints of different parameters $n$ and Fourier series. The symbol signal obtained by the numerical solution is also an approximate solution, but it gradually approaches the true solution by increasing $m$. Additionally, by analyzing the index of the mean square error of the optimal symbol signal with the number of adjacent Fourier series items $m$ and $m-1$, it is found that the value of $m$ increases to a certain value and is infinitely close to the exact solution. The two time-domain waveforms are compared and analyzed under the same parameters of $n=2$ and $\mathrm{PF}=1.58$ to make the characteristics between the analytical solution and the numerical solution more intuitive. The above series of comparative analysis results show that the optimal symbol signal obtained by the numerical function solution by establishing a generic function is more suitable for modern digital communication systems.

\section{Optimal Generic Function}

2.1. Minimum Out-of-Band Energy Radiation Criteria. To obtain a quadrature modulated baseband symbol signal that has a spectral fast rolling-off characteristic, the minimum out-of-band energy radiation criterion is introduced to establish a mathematical generic function model [22], which can be expressed as follows:

$$
J=\frac{1}{2 \pi} \int_{-\infty}^{+\infty} g(\omega)|S(\omega)|^{2} \mathrm{~d} \omega
$$

where $g(\omega)$ is a function determining the rolling-off speed of the energy spectrum $|S(\omega)|^{2}$ of the baseband symbol signal $a(t)$, and $S(\omega)$ is the spectral density function of the signal $a(t)$. The length of $a(t)$ is set to $T$, and it is also assumed that $a(t)$ is an even function in the interval $[-T / 2$, $T / 2$ ], where $T$ is the unit bit symbol signal length.

The form of $g(\omega)$ is the ascending function $g(\omega)=$ $\omega^{2 n}, n=1,2,3, \ldots$. The value of $n$ depends on the extent of the out-of-band energy radiation to be suppressed. To make the generic integral $J$ convergent and integrable, the falling speed of $|S(\omega)|^{2}$ must be greater than the rising speed of the rising function $g(\omega)$, and thereby the purpose of limiting the energy spectrum rolling-off speed of the signal $a(t)$ is achieved. Then, formula (1) can be written after a series of derivations as follows:

$$
J=(-1)^{n} \int_{-T / 2}^{T / 2} a(t) a^{(2 n)}(t) \mathrm{d} t,
$$

where $a^{2 n}(t)$ is the $2 n$-order derivative of the symbol signal $a(t)$. In this way, the minimum out-of-band energy radiation criterion can be summarized as the problem of solving the baseband symbol function under the condition that the mathematical generic function $J$ is guaranteed to be the minimum.

2.2. Limitations of the General Function. The above generic function $J$ needs to be attached with restrictions to make the obtained signal have the required characteristics. There are three restrictions; the first is the energy limitation of single symbol signal:

$$
E=\int_{-T / 2}^{T / 2} a^{2}(t) \mathrm{d} t
$$

The energy limitation is the important constraint for the generic function model. Recently, the energy efficient schemes, such as spatial modulation [23] or quadrature spatial modulation [24], are proposed to relieve this problem.

Then, there is the boundary condition limitation, and the signal with the symbol length $T$ is required to have a value of 0 at the time end $\pm T / 2$. Moreover, its $n-1$ derivative has a value of $\pm T / 2$ at the end of time, which can be expressed as follows:

$$
a\left(\frac{ \pm T}{2}\right)=a^{\prime}\left(\frac{ \pm T}{2}\right)=\cdots=a^{(n-1)}\left(\frac{ \pm T}{2}\right) .
$$

Finally, the peak-to-average ratio limit of a single symbol signal, and the concept of a single symbol signal peak-toaverage ratio limit is introduced as follows:

$$
\mathrm{PF}=\frac{a_{\max }}{\sqrt{(1 / T) \int_{-T / 2}^{T / 2} a^{2}(t) \mathrm{d} t}} .
$$

To simplify the calculation process, the following equation is used instead of the peak-to-average ratio constraint:

$$
\alpha=\frac{a_{\max }}{(1 / T) \int_{-T / 2}^{T / 2} a^{2}(t) \mathrm{d} t} .
$$

Formula (6) shows a limit on the peak-to-average ratio, and it can be expressed as follows: 


$$
\int_{-T / 2}^{T / 2}\left(1-\frac{T}{\alpha} \cdot \delta(t)\right) a(t) \mathrm{d} t=0 .
$$

According to the theory of subdivision theory, the Lagrange function can be expressed as follows:

$$
H=(-1)^{n} a(t) a^{(2 n)}(t)-\lambda a^{2}(t)-\mu a(t)\left(1-\frac{T}{\alpha} \cdot \delta(t)\right),
$$

where $\lambda$ and $\mu$ are Lagrange operators.

In this way, the minimum value of the generic function $J$ that adds a single symbol signal energy and a peak-to-average ratio constraint condition should satisfy the following Euler equation:

$$
\frac{\partial H}{\partial a}+\frac{\mathrm{d}^{2 n}}{\mathrm{~d} t^{(2 n)}}\left(\frac{\partial H}{\partial a^{(2 n)}}\right)=0
$$

Therefore, the desired symbol expression can be obtained by solving the following differential equation:

$$
(-1)^{n} a(t) a^{(2 n)}(t)-\lambda a(t)-\mu a(t)\left(1-\frac{T}{\alpha} \cdot \delta(t)\right)=0 .
$$

\section{Analytic Solution of the Optimal General Function Model}

3.1. Analytic Solution of the Optimal General Function at $n=1$. When $n=1$, there are $g(\omega)=\omega^{2}$, and formula (10) can be converted to the following form:

$$
a_{1}(t) a_{1}^{(2)}(t)-\lambda a_{1}(t)-\frac{\mu}{2}\left(1-\frac{T}{\alpha} \cdot \delta(t)\right)=0,
$$

where $\lambda$ and $\mu$ are Lagrange operators and $T$ is the symbol signal length.

Boundary conditions can be expressed as follows:

$$
a_{1}\left(\frac{ \pm T}{2}\right)=0
$$

Through the peak-to-average ratio and energy limit conditions, the optimal symbol can be expressed as follows [25]:

$$
\begin{aligned}
a_{1}(t)= & \frac{E^{1 / 2}}{\left(\left(2 A \cos ^{2}(A)-3 \sin (A) \cos (A)+A\right) /\left(2 A \cos ^{2}(A)\right)\right)^{1 / 2}} \\
& \cdot\left(1-\frac{\cos (2 A t / T)}{\cos (A)}\right),
\end{aligned}
$$

where $A$ is a constant.

By limiting the peak-to-average ratio, the following can be obtained:

$$
\begin{aligned}
\alpha(A) & =\frac{A \cos (A)-A}{A \cos (A)-A \sin (A)} ; \\
\mathrm{PF} & =\sqrt{\frac{T}{E} C_{a 1}(A)\left(1-\frac{1}{\cos (A)}\right)},
\end{aligned}
$$

where $C_{a 1}$ is a constant depending on $A$.

The waveforms of the optimized symbol signals $a_{1}(t)$ corresponding to different PF values and the waveforms of the energy spectral density are shown in Figure 1.

In Figure 1, $a_{11}(t)$ corresponds to $\mathrm{PF}=1.42(A=1.6)$, $a_{12}(t)$ corresponds to $\mathrm{PF}=1.5(A=2.5)$, and $a_{13}(t)$ corresponds to $\mathrm{PF}=1.63(A=3.14)$.

It can be seen from Figure 1 that the signal power spectral density decays the fastest when $P F=1.63$. The peakto-average ratio of the symbol signal has a certain influence on the time-frequency characteristics of the signal, as shown from the time-frequency characteristics of the optimized signal. As the PF value increases, the signal becomes smoother at the time end $\pm T / 2$, and the out-of-band energy spectral density decays faster. However, when the PF value is higher than 1.63, the optimization signal appears to oscillate around the time axis $t$.

3.2. Analytic Solution of the Optimal General Function at $n=2$. The process of solving the analytical solution of the generalized function at $n=2$ is somewhat similar to the process of solving $n=1$. The difference is that the difficulty of the solution process is increased because of the need to solve higher-order differential equations when $n=2: g(\omega)=\omega^{4}$.

After appending a single symbol signal energy limit and a symbol signal peak-to-average power ratio, the solution of the optimized signal $a_{2}(t)$ can be obtained by solving the following equation:

$$
a_{2}(t) a_{2}^{(4)}(t)-\lambda a_{2}(t)-\frac{\mu}{2}\left(1-\frac{T}{\alpha} \cdot \delta(t)\right)=0,
$$

where $\lambda$ and $\mu$ are Lagrange operators, $T$ is the symbol signal length, and $a_{2}^{(4)}(t)$ is the fourth derivative of $a_{2}(t)$. The following boundary conditions should also be considered to solve this differential equation:

$$
a_{2}\left(\frac{ \pm T}{2}\right)=a_{2}^{\prime}\left(\frac{ \pm T}{2}\right)=0
$$

Therefore, the following expression of $a_{2}(t)$ can be obtained [25]:

$$
a_{2}(t)=C_{a 2}\left(1-\frac{\cos \left(2 A_{2} t / T\right) \cdot \operatorname{sh}\left(A_{2}\right)+\operatorname{ch}\left(2 A_{2} t / T\right) \cdot \sin \left(A_{2}\right)}{\cos \left(A_{2}\right) \cdot \operatorname{sh}\left(A_{2}\right)+\operatorname{ch}\left(A_{2}\right) \cdot \sin \left(A_{2}\right)}\right) \text {. }
$$

The spectral function of the optimized symbol signal $a_{2}(t)$ can be easily obtained according to the Fourier transform and the symmetry of signal $a_{2}(t)$. The waveforms of the optimized symbol signals $a_{2}(t)$ corresponding to different PF values and the waveforms of the energy spectral density are shown in Figure 2.

As shown in Figure 2, when the peak-to-average power ratio of signal $a_{2}(t)$ is maximized, that is, $\mathrm{PF}=1.74$ $\left(A_{2}=3.926\right)$, its energy spectral density decays faster. Therefore, when $\mathrm{PF}=1.74\left(A_{2}=3.926\right)$, the resulting signal $a_{2}(t)$ is the optimal signal form when $n=2$.

The above analytical solution process significantly simplifies the partial solution process, and the obtained 

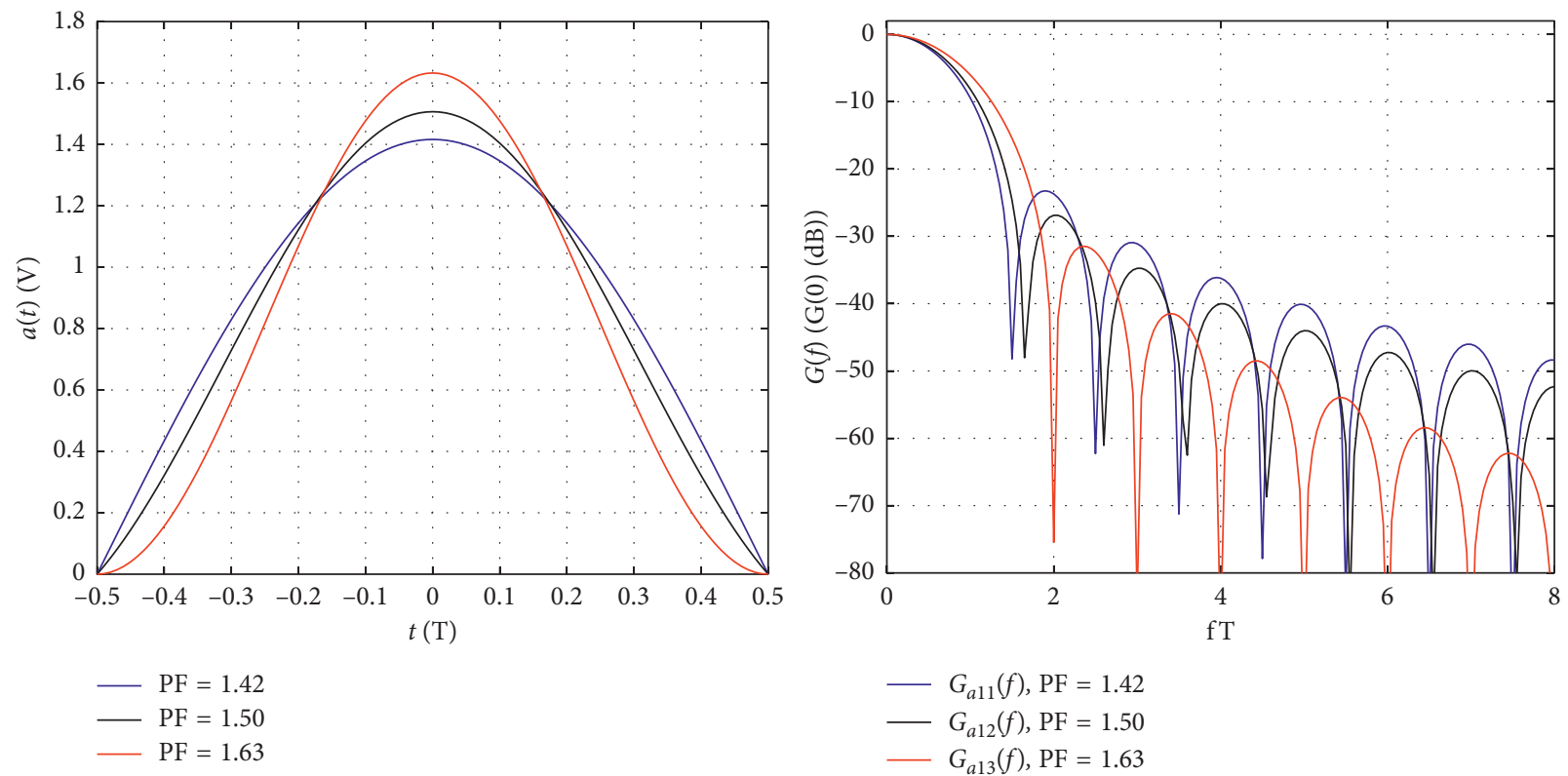

Figure 1: Waveforms and spectral density of the optimized symbol signals $a_{1}(t)$ for different PF values.
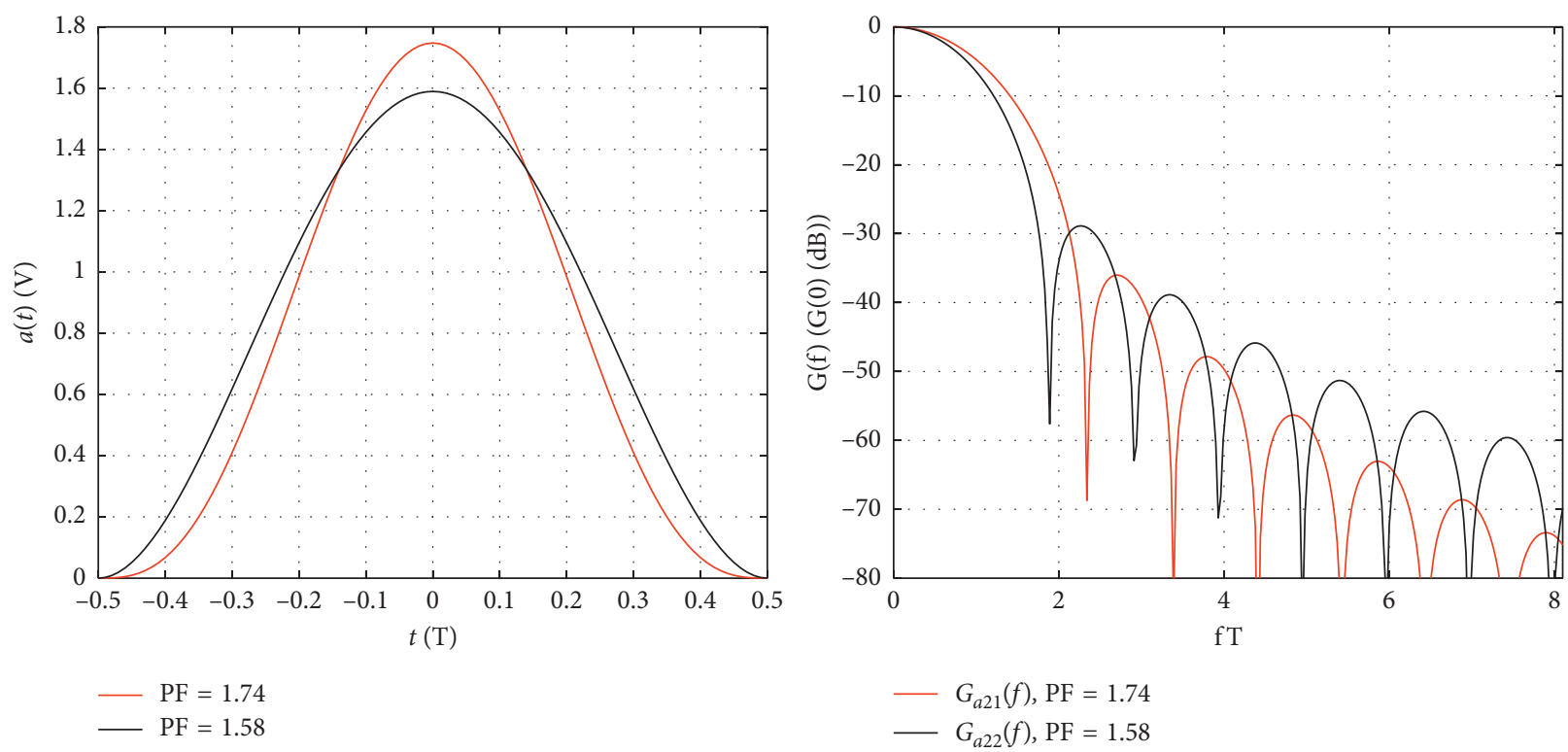

FIgURE 2: Waveforms and spectral density of the optimal symbol signals $a_{2}(t)$ for different PF values.

symbol signal is an approximate solution of the optimal symbol signal to be obtained by initially establishing the constraint added in the generic function model. In addition, the corresponding order of Lagrange differential equation increases by a multiple of 2 when the parameter $n$ increases, and the solution is extremely complicated or even impossible to solve. Therefore, the numerical method is chosen instead of the analytical method.

\section{Numerical Solution of the Optimal Generic Function Model}

4.1. Numerical Solution Model of the Optimal Generic Function Model. It can be seen from the solution process of the analytical solution of the optimal generic function model that when $n$ takes a higher value, its solution process is very difficult. Moreover, only the single symbol signal energy limit, the symbol signal peak-to-average power ratio, and the boundary condition limit are added in the optimal generic model. To broaden the application field of the method, this is a practical and feasible way to introduce the complete orthogonal set series by a numerical method to realize the approximate solution of the optimal generic model.

The additional constraints, that is, the single symbol signal energy limit condition, the single symbol signal peakto-average ratio limit condition, and the boundary constraint condition of the optimal generic model numerical solution process are discussed below. 
A generic function that adds a single symbol signal energy limit condition and a single symbol signal peak-toaverage limit condition can be expressed as follows:

$$
\begin{aligned}
H & =J+\lambda\left(\int_{-T / 2}^{T / 2} a^{2}(t) \mathrm{d} t-E\right)+\mu\left(a(0)-\mathrm{PF} \sqrt{\frac{E}{T}}\right), \\
J & =(-1)^{n} \int_{-T / 2}^{T / 2} a(t) a^{2 n}(t) \mathrm{d} t,
\end{aligned}
$$

where $a^{2 n}(t)$ is the $2 n$-order derivative of the symbol signal $a(t)$.

The energy of a single symbol signal can be expressed as follows:

$$
E=\int_{-T / 2}^{T / 2} a^{2}(t) \mathrm{d} t
$$

The peak-to-average ratio of a single symbol signal can be expressed as follows:

$$
\mathrm{PF}=\frac{a_{\max }}{\sqrt{(1 / T) \int_{-T / 2}^{T / 2} a^{2}(t) \mathrm{d} t}}=\frac{a_{0}}{\sqrt{(1 / T) \int_{-T / 2}^{T / 2} a^{2}(t) \mathrm{d} t}}=\frac{a_{0}}{\sqrt{(E / T)}} .
$$

There is the boundary condition limitation, and the signal with the symbol length $T$ is required to have a value of 0 at $\pm T / 2$. Moreover, its $n-1$ derivative has a value of $\pm T / 2$ at the end time, which can be expressed as follows:

$$
a\left(\frac{ \pm T}{2}\right)=a^{\prime}\left(\frac{ \pm T}{2}\right)=\cdots=a^{(n-1)}\left(\frac{ \pm T}{2}\right)=0 .
$$

Considering that the desired optimal function has an even function characteristic, it can be expressed as a Fourier series as follows:

$$
a(t)=\frac{a_{0}}{2}+\sum_{k=1}^{m} a_{k} \cos \left(\frac{2 \pi}{T} k t\right) .
$$

The coefficients of the Fourier series are determined by the following formulas:

$$
\begin{aligned}
& a_{0}=\frac{2}{T} \int_{-T / 2}^{T / 2} a(t) \mathrm{d} t \\
& a_{k}=\frac{2}{T} \int_{-T / 2}^{T / 2} a(t) \cos \left(\frac{2 \pi k t}{T}\right) \mathrm{d} t .
\end{aligned}
$$

Equation (22) can be substituted into equation (19), but the coefficients $a_{0}, a_{1}, \ldots, a_{k}$ of the Fourier series and the Lagrange coefficients $\lambda$ and $\mu$ are unknown for a specific symbol signal energy $E$ and symbol signal length $T$ in the generic function. The Fourier series expression of the optimal symbol signal can be obtained if the value of the Fourier series coefficients $a_{0}, a_{1}, \ldots, a_{k}$ are found and then substituted into equation (22). Its accuracy depends on the number of terms of the Fourier series. The more items there are, the closer the numerical solution will be to the analytical solution of $a(t)$.

The minimum value of $H$ that should satisfy the boundary constraints and the Lagrange equations are as follows:

$$
\begin{aligned}
& \frac{\partial H}{\partial a_{k}}=0, \\
& \frac{\partial H}{\partial \lambda}=0 ; \\
& \frac{\partial H}{\partial \mu}=0, \quad k=1,2, \ldots, m ;
\end{aligned}
$$

\subsection{Numerical Solution of the Optimal Generic Function} Model with Different Values of Parameters $n$ and PF. The Fourier series term number $m$ takes different values to achieve the optimal solution of the generic function when $n=2$ and $E=1$. At this time, the boundary constraint condition is $a( \pm T / 2)=a^{\prime}( \pm T / 2)=0$, and the single symbol signal peak average ratio constraint condition is $\mathrm{PF}=1.58$. MATLAB can be used to realize the solution process, and the results are shown in Table 1.

The Fourier series term number $m$ takes different values to achieve the optimal solution of the generic function when $n=4$ and $E=1$. At this time, the boundary constraint condition is $a( \pm T / 2)=a^{\prime}( \pm T / 2)=a^{\prime \prime}( \pm T / 2)=a^{\prime \prime \prime}$ $( \pm T / 2)=0$, and the single symbol signal peak average ratio constraint condition is $\mathrm{PF}=1.85$. MATLAB can be used to realize the solution process, and the results are shown in Table 2.

The Fourier series term number $m$ takes different values to achieve the optimal solution of the generic function when $n=6$ and $E=1$. At this time, the boundary constraint condition is $a( \pm T / 2)=a^{\prime}( \pm T / 2)=\cdots=a^{(5)}( \pm T / 2)=0$, and the single symbol signal peak average ratio constraint condition is $P F=2.02$. MATLAB can be used to realize the solution process, and the results are shown in Table 3.

To compare the influence of the term $m$ of the Fourier series on the optimal signal accuracy, the concept of mean square error of an optimized symbol signal with adjacent Fourier series number $m$ and $m-1$ is introduced, which can be expressed as follows:

$$
\varepsilon_{m}=\overline{\left|a_{m}(t)-a_{m-1}(t)\right|^{2}},
$$

where $a_{m}(t)$ and $a_{m-1}(t)$, respectively, represent optimal symbol signals of which the number of Fourier series items is $m$ and $m-1$.

According to the data in Tables 1-3 and the concept of mean square error of the optimized symbol signal with adjacent Fourier series terms $m$ and $m-1$ introduced above, the mean square error curve of the optimized symbol signals has the number of adjacent Fourier series terms $m$ and $m-1$ when the generic function parameter $n$ takes different values, as shown in Figure 3.

From the data in Figure 3, it can be seen that as the value of $m$ increases, the number of items in the Fourier series increases continuously, and when the parameter $n$ is constant, the gap between the adjacent optimal symbol signals is rapidly reduced and the order of magnitude can reach $10^{-5}$. The slope between the two adjacent points drops sharply, as 
TABLE 1: When $n$ is 2 , the coefficient of the Fourier series number $m$ is different.

\begin{tabular}{rcccccrrr}
\hline$m$ & $a_{0}$ & $a_{1}$ & $a_{2}$ & $a_{3}$ & $a_{4}$ & $a_{5}$ & $a_{6}$ \\
\hline 3 & 1.6633 & 0.7842 & -0.0415 & 0.0059 & - & - & - & - \\
4 & 1.6638 & 0.7838 & -0.0396 & 0.0061 & -0.0024 & - & - \\
5 & 1.6643 & 0.7832 & -0.0397 & 0.0061 & -0.0024 & 0.0008 & - \\
6 & 1.6643 & 0.7832 & -0.0391 & 0.0061 & -0.0024 & 0.0008, & -0.0005 \\
7 & 1.6646 & 0.7829 & -0.0393 & 0.0061 & -0.0024 & 0.0008 & -0.0005 & - \\
\hline
\end{tabular}

TABLE 2: When $n$ is 4 , the coefficient of the Fourier series number $m$ is different.

\begin{tabular}{ccccccccc}
\hline$m$ & $a_{0}$ & $a_{1}$ & $a_{2}$ & $a_{3}$ & $a_{4}$ & $a_{5}$ & $a_{6}$ \\
\hline 3 & 1.4601 & 0.9443 & 0.1968 & -0.0174 & - & - & - & - \\
4 & 1.4686 & 0.9395 & 0.1858 & -0.0163 & 0.0031 & - & - \\
5 & 1.4765 & 0.9384 & 0.1796 & -0.0167 & 0.0031 & -0.0008 & - \\
6 & 1.4795 & 0.9373 & 0.1765 & -0.0168 & 0.0031 & -0.0008 & 0.0003 \\
7 & 1.4810 & 0.9369 & 0.1754 & -0.0167 & 0.0031 & -0.0008 & 0.0003 & -0.0001 \\
\hline
\end{tabular}

TABLE 3: When $n$ is 6 , the coefficient of the Fourier series number $m$ is different.

\begin{tabular}{ccccccccc}
\hline$m$ & $a_{0}$ & $a_{1}$ & $a_{2}$ & $a_{3}$ & $a_{4}$ & $a_{5}$ & $a_{6}$ \\
\hline 4 & 1.3359 & 0.9811 & 0.3591 & 0.0400 & -0.0060 & - & - & - \\
5 & 1.3478 & 0.9799 & 0.3448 & 0.0328 & -0.0050 & 0.0010 & - \\
6 & 1.3565 & 0.9809 & 0.3382 & 0.0299 & -0.0046 & 0.0009 & -0.0002 \\
7 & 1.3596 & 0.9801 & 0.3341 & 0.0283 & -0.0043 & 0.0008 & -0.0002 \\
\hline
\end{tabular}

shown in the figure. When the value of $m$ reaches a certain value, the difference between adjacent ones can be neglected. If $n=2, m=6$, and $\mathrm{PF}=1.58$, then the mean square error is $2.703 e-07, n=2, m=7$; when $\mathrm{PF}=1.58$, the mean square error is $9.898 e-08$. This phenomenon proves that the value of $m$ does not have to be large to be considered as meeting the optimal solution required by the established generic function model and the constraint conditions. This is incomparable to the analytical solution process.

4.3. Normalized Energy Spectral Density Analysis of the Optimized Symbol Signals under Different Parameters. A symbol signal with excellent spectral characteristics is obtained by introducing the minimum out-of-band energy radiation criterion and optimizing the baseband symbol signal by establishing a generic function model. Therefore, it is necessary to examine the influence of the number of items $m$ of the Fourier series on the spectral characteristics of the signal.

Figure 4 shows the waveform diagram of the optimal symbol signal with $n=2, n=4$, and $n=6$ with adjacent Fourier series number terms $m=6$ and $m=7$. Their mean square error is no more than $0.01 \%$. The normalized energy spectral density curve of the above signal is also shown in Figure 5, where Figure 5(a) shows the normalized energy spectral density of the baseband symbol signal at $\mathrm{PF}=1.58$, $n=2, m=6$, and $m=7$; Figure 5 (b) shows the normalized energy spectral density of the baseband symbol signal at $\mathrm{PF}=1.85, n=4, m=6$, and $m=7$; Figure 5(c) shows the normalized energy spectral density of the baseband symbol signal at $\mathrm{PF}=2.02, n=6, m=6$, and $m=7$; and Figure $5(\mathrm{~d})$

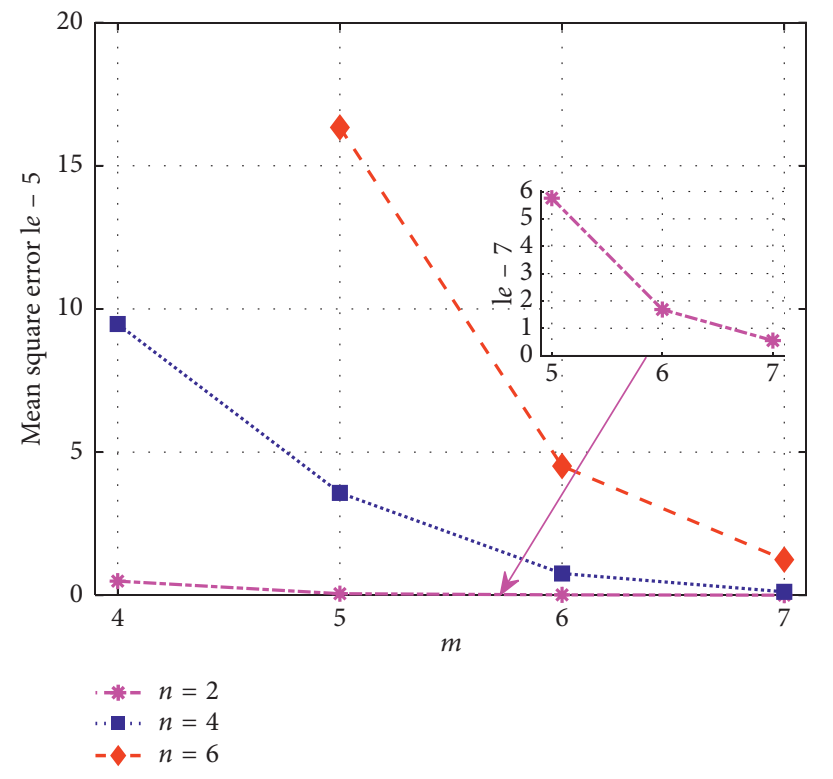

FIgURE 3: Mean variance curve of the optimal symbol signals with adjacent Fourier series numbers $m$ and $m-1$ when $n$ takes different values.

shows the normalized spectral density when the length of the ascending cosine signal and the cosine signal is 1 and the normalized spectral density of baseband symbol signals when $\mathrm{PF}=2.02, n=6$, and $m=7$.

The time-domain diagram given in Figure 4 is smoother at the boundary as the value of $n$ increases. This property shows that the constraints at the boundary play a significant role, and it also gives the optimal symbol signal a more 


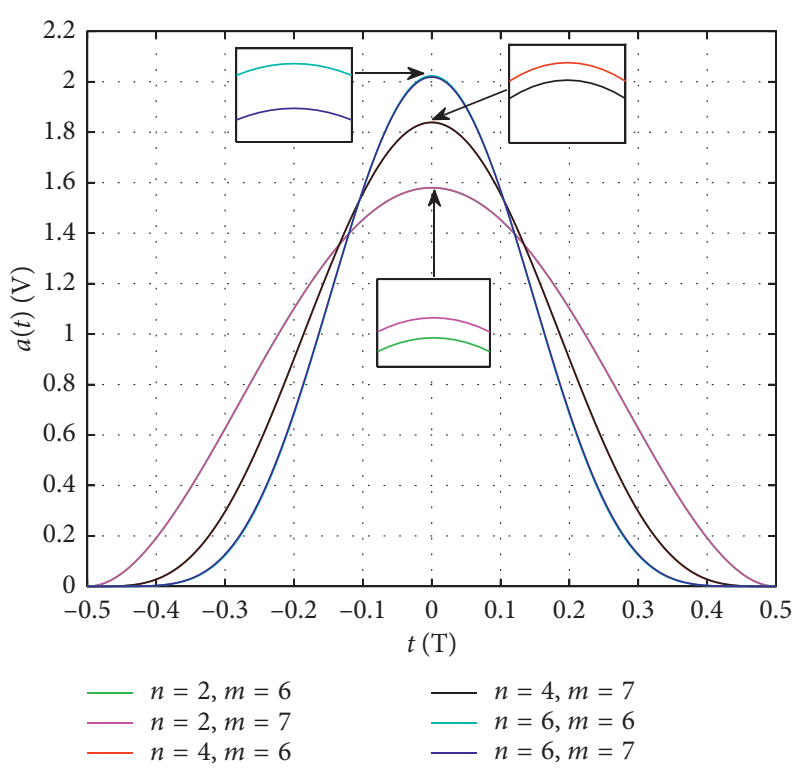

FIGURE $4: n=2, n=4$, and $n=6$, and the optimal symbol signal waveforms with the adjacent Fourier series number of terms $m=6$ and $m=7$.

excellent property in the frequency domain. The numerical solutions solved for different parameters are analyzed for their different properties in the frequency domain. When $\mathrm{PF}=1.58, n=2, m=6$, and $m=7$, the spectral density of the optimized symbol signal has a main lobe width of $1.865 \mathrm{fT}$; when $\mathrm{PF}=1.85, n=4, m=6$, and $m=7$, the spectral density of the optimized symbol signal has a main lobe width of $2.636 \mathrm{fT}$; when $\mathrm{PF}=2.02, n=6, m=6$, and $m=7$, the spectral density of the optimized symbol signal has a main lobe width of $3.387 \mathrm{fT}$. As the value of $n$ increases, the main spectral width of the spectral density of the optimal symbol signal increases significantly. In addition, a small increase in the width of the main lobe also causes the energy of the signal to concentrate more on the main lobe. This effectively reduces the out-of-band energy radiation, which is of great significance to the practical application of modern digital communication transmission. As the value of $n$ increases, the energy of the optimal symbol signal is more concentrated in the main lobe, and the out-of-band radiation will be further reduced. That is to say, the noise interference received can be effectively suppressed by other means when the communication system transmits. From the perspective of hardware implementation, the numerical solution is easier to implement in hardware systems like FPGA, and it is more in line with the current trend of digital communication.

The value of $n$ depends on the degree of energy radiation outside the frequency band to be suppressed. In other words, the value of $n$ is a parameter that has the greatest influence on the waveform. As the value of $n$ increases, the peak value of the symbol waveform will also increase, and the symbol waveform will decrease faster. In fact, the numerical solution is the approximate solution of the ideal solution. When $n=2$, there is also a difference between the energy spectrum density. As the $n$ value increases, the difference will be amplified. However, this difference basically occurs below
$-60 \mathrm{~dB}$, and the spectrum of the communication system is basically not taken below $-60 \mathrm{~dB}$, so this difference does not affect the performance of the communication system.

The normalized energy spectral density curve of the raised cosine signal and the cosine signal of the empirical signals of Figure 5(d) can be compared with the normalized energy spectrum density of the baseband symbol signal when $\mathrm{PF}=2.02, n=6$, and $m=7$ to more intuitively analyze the good frequency-domain properties of the baseband symbol signals solved by the generic function model. Calculated by $-40 \mathrm{~dB}, \mathrm{PF}=2.02, n=6$, and $m=7$, the normalized energy spectral density occupies a bandwidth of $3.148 \mathrm{fT}$. The normalized energy spectral density of the cosine and raised cosine signals is $5.029 \mathrm{fT}$ and $18.6 \mathrm{fT}$, respectively. When $n=6$ and $m=7$, the frequency band utilization of the baseband symbol signal is 6.19 times higher than that of the raised cosine signal. Calculated by $-60 \mathrm{~dB}$, $\mathrm{PF}=2.02, n=6$, and $m=7$, the normalized energy spectral density occupies a bandwidth of $4.345 \mathrm{fT}$. The normalized energy spectral densities of the cosine and raised cosine signals are $16 \mathrm{fT}$ and $194.5 \mathrm{fT}$, respectively. When $n=6$ and $m=7$, the frequency band utilization of the baseband symbol signal is 3.68 times and 44.76 times higher than that of the cosine signal and the raised cosine signal. The comparison above shows that the numerically resolved baseband symbol signal obtained by establishing the optimal generic function is more suitable for modern wireless communication transmission. For example, it is more suitable for a baseband symbol signal or the like as a continuous phase modulation (CPM) signal.

The mean deviation of normalized energy spectral density absolute value of optimized symbol signal with adjacent Fourier series terms $m$ and $m-1$ when $n$ takes the same value is introduced below. It can be expressed as follows:

$$
\varepsilon_{f}(m)=\overline{\left|\frac{G_{m}(f)}{G_{m}(0)}-\frac{G_{m-1}(f)}{G_{m-1}(0)}\right|},
$$

where $G_{m}(f)$ is the energy spectral density of the optimized symbol signal when the number of terms of the Fourier series is $m$.

Figure 6 shows the normalized energy spectral densities of the difference between the corresponding frequency points of the adjacent frequency range of $3 \mathrm{fT}-4 \mathrm{fT}$ when the parameters $n$ are taken as 2, 4, and 6, respectively. There is an average deviation of the absolute values.

Figure 6 shows the normalized energy spectral density when the Fourier series $m$ are taken as $4,5,6$, and 7 . A rapid decreasing trend with the increase of $m$ value is shown in the average deviation of the absolute value of the normalized energy spectral density of the difference between the corresponding frequency points in the adjacent frequency range of $3 \mathrm{fT}-4 \mathrm{fT}$. When $n=2$, the average deviation of the absolute value of the normalized energy spectral density of the difference between the corresponding frequency points in the adjacent frequency range of $3 \mathrm{fT}-4 \mathrm{fT}$ is $0.01 \%$ or less, and its energy spectral density meets the requirements for out-of-band radiation levels. This shows that the average deviation of the absolute value of the normalized energy 

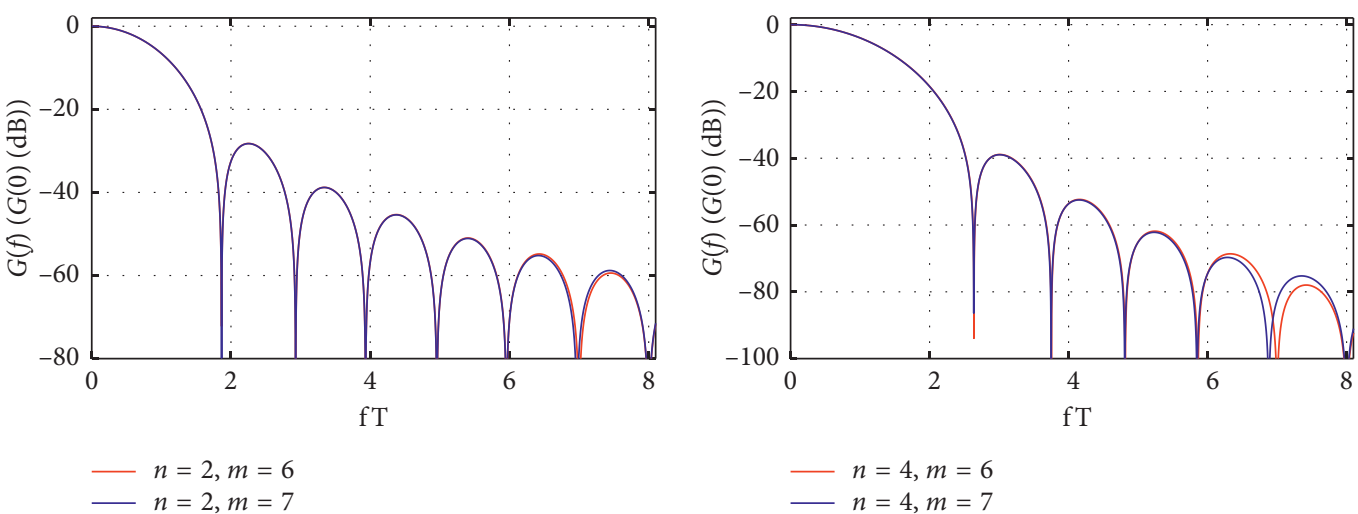

(a)
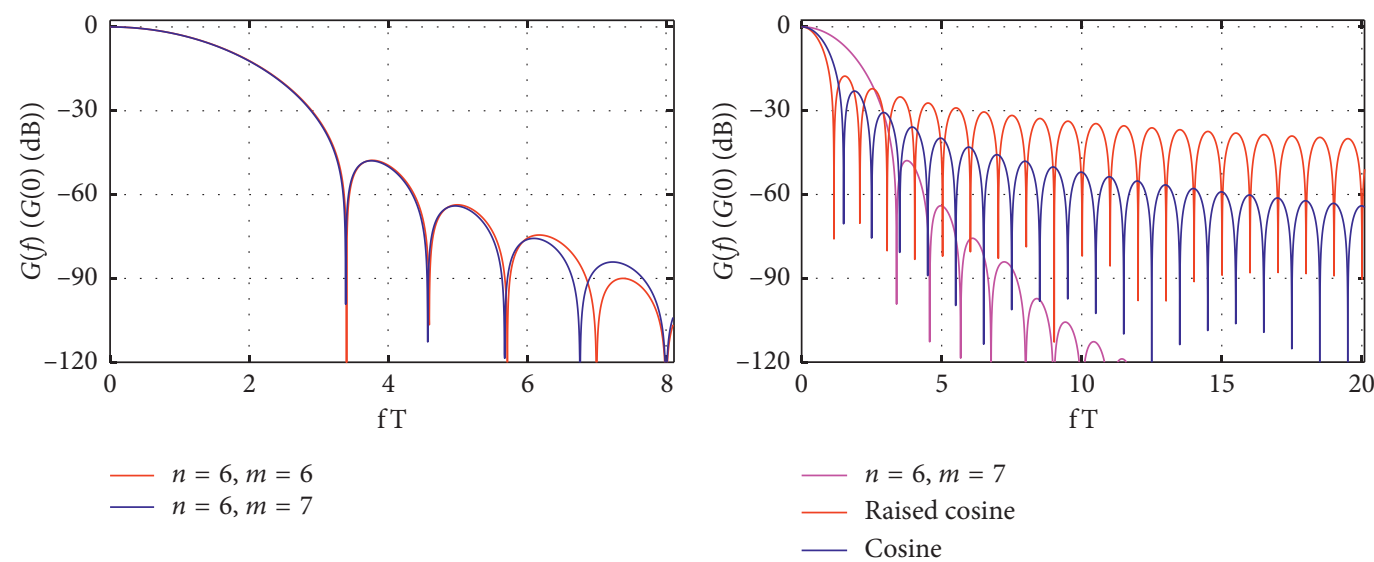

(c)

(d)

FIGURE 5: (a) The normalized energy spectral density of the baseband symbol signal at $\mathrm{PF}=1.58, n=2, m=6$, and $m=7$; (b) the normalized energy spectral density of the baseband symbol signal at $\mathrm{PF}=1.85, n=4, m=6$, and $m=7$; (c) the normalized energy spectral density of the baseband symbol signal at $\mathrm{PF}=2.02, n=6, m=6$, and $m=7$; and (d) the normalized spectral density when the length of the ascending cosine signal and cosine signal is 1 and the normalized spectral density of baseband symbol signals when $\mathrm{PF}=2.02, n=6$, and $m=7$.

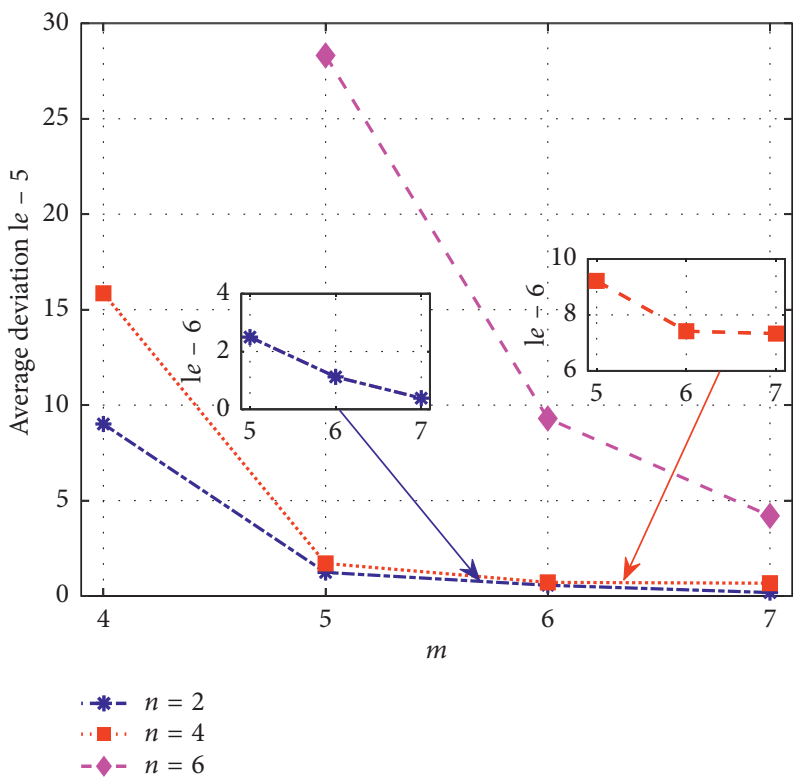

FIGURE 6: The normalized energy spectral density of the difference between the corresponding frequency points of the adjacent frequency range of $3 \mathrm{fT}-4 \mathrm{fT}$ when the parameters $n$ are taken as 2,4 , and 6 , respectively. There is an average deviation of the absolute values. 

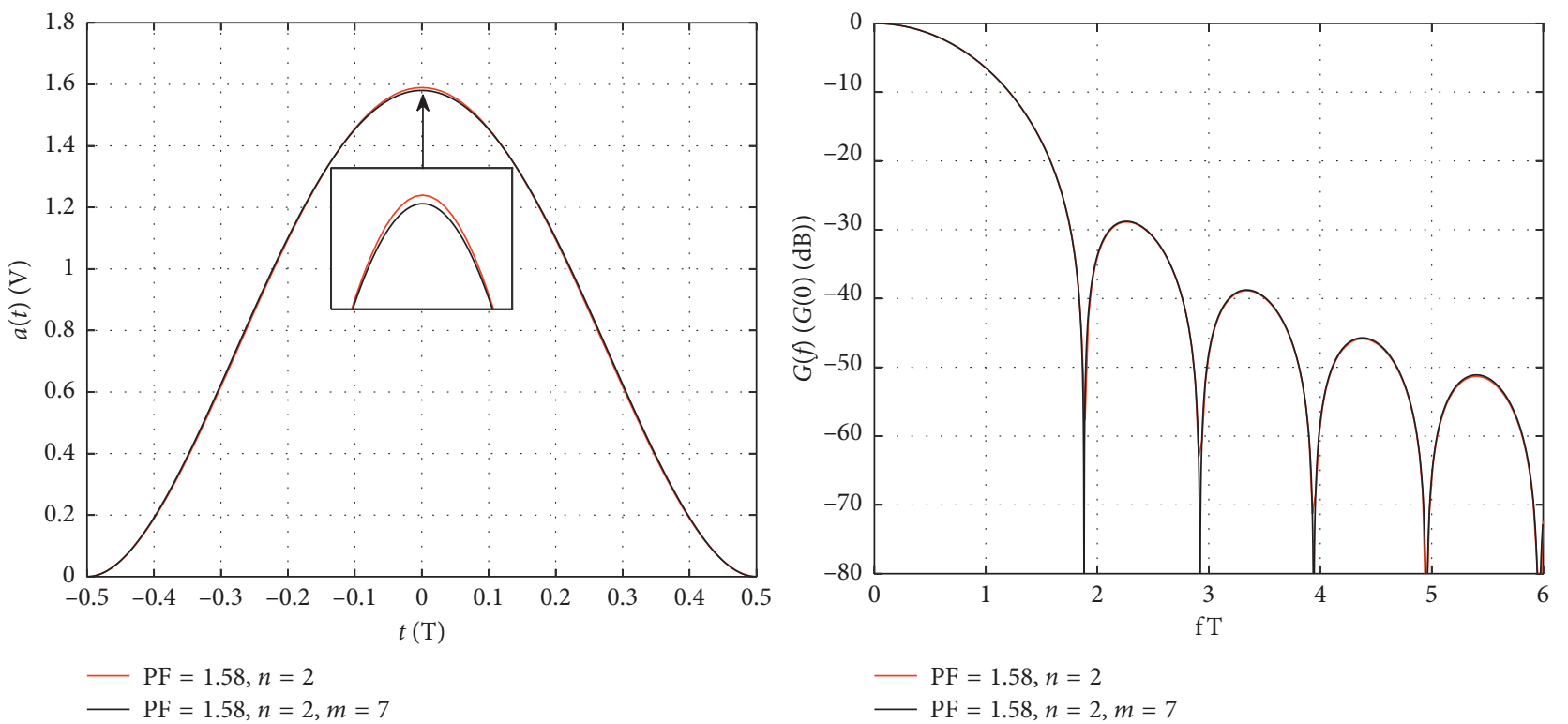

FIGURE 7: Comparison of time-domain graphs and normalized energy spectrum density of numerical solutions for $\mathrm{PF}=1.58$ and $n=2$ and $\mathrm{PF}=1.58, n=2$, and $m=7$.

spectral density corresponding to the difference between subsequent adjacent intervals is further reduced. This phenomenon is of great significance in communication transmission.

\subsection{Accuracy Comparison between the Analytical Solution and} the Numerical Solution When $P F=1.58$ and $n=2$. The analytical solution when $\mathrm{PF}=1.58$ and $n=2$ is compared with the numerical solution when $\mathrm{PF}=1.58, n=2$, and $m=7$ to describe the exact relationship between numerical solutions and exact solutions, as shown in Figure 7. It can be seen intuitively from Figure 7 that the analytical solution of $\mathrm{PF}=1.58$ and $n=2$ and the numerical solution of $\mathrm{PF}=1.58$ and $n=2$, and $m=7$ are basically completely coincident. In terms of specific values, the mean square deviation calculated between them is $5.5497 e-05$.

In the analysis of the aforementioned error causes, first of all, in the process of solving the analytical solution, the simplification of the peak-to-average ratio constraints simplifies the process, which makes the calculation process simpler but also reasonable. Secondly, the numerical solution itself is an approximate solution. The accuracy will be infinitely close to the true solution as the number of terms of the Fourier series increases. Therefore, we think that the numerical solution method can replace the analytical solution method with the complicated calculation process.

\section{Conclusions}

In this paper, a generic model of optimal symbol signal is established based on the minimum out-of-band energy radiation criterion. The boundary function and the energy limit of the symbol signal are imposed on the generic function model. The optimal symbol signals are obtained by the analytical method and the numerical method. The energy of the symbol signal obtained by establishing the optimal generic function is more concentrated, and the out-of-band radiation is smaller. However, the partial solution process is significantly simplified by the analytical solution. The obtained symbol signal is an approximate solution of the optimized symbol signal to be obtained by initially establishing the constraint added in the generic function model. For the numerical solution, the concept of mean square error of the optimal symbol signal with the number of adjacent Fourier series terms $m$ and $m-1$ is introduced. An analysis of the time-domain properties of the symbol signal shows that as the value of $m$ increases, the number of items of the Fourier series increases continuously, and the difference between the adjacent optimized symbol signals is rapidly reduced when parameter $n$ is unchanged. The order of magnitude can reach $10^{-5}$. It is concluded that the value of $m$ does not have to be large enough to be consistent with the established generic function model and the optimal solution that the constraint requires. This excellent property is unmatched by the analytical solution. The normalized energy spectral density, calculated by $-60 \mathrm{~dB}, \mathrm{PF}=2.02, n=6$, and $m=7$, occupies a bandwidth of $4.345 \mathrm{fT}$. The normalized energy spectral densities of the cosine and raised cosine signals are $16 \mathrm{fT}$ and $194.5 \mathrm{fT}$, respectively. When $n=6$ and $m=7$, the frequency band utilization of the baseband symbol signal is 3.68 times and 44.76 times higher than that of the cosine signal and the raised cosine signal. The above comparison shows that the numerically resolved baseband symbol signal obtained by establishing the optimal generic function is more suitable for modern wireless communication transmission. For example, it is more suitable for a baseband symbol signal or the like as a continuous phase modulation (CPM) signal. The mean deviation of normalized energy spectral density absolute value of optimal symbol 
signal with adjacent Fourier series terms $m$ and $m-1$ in the adjacent frequency interval is introduced. When $n=2$, the average deviation of the absolute value of the normalized energy spectral density of the difference between the corresponding frequency points in the adjacent frequency range of $3 \mathrm{fT}-4 \mathrm{fT}$ is $0.01 \%$ or less, and its energy spectral density meets the requirements for out-of-band radiation levels. As shown from the above-stated analysis, the average deviation of the absolute value of the normalized energy spectral density corresponding to the difference between subsequent adjacent intervals is further reduced. This phenomenon is of great significance for communication transmission. To describe the exact relationship and more intuitively compare the difference between the optimized symbol signal obtained by the numerical solution and the optimized symbol signal obtained by the analytical solution, the analytical solution with $\mathrm{PF}=1.58$ and $n=2$ is compared with the numerical solution for $\mathrm{PF}=1.58, n=2$, and $m=7$. The results show that with the increase in the number of Fourier series, the numerical solution can be used to replace the complex analytical solution of the computational process.

\section{Data Availability}

The data used to support the findings of this study are available from the corresponding author upon request.

\section{Conflicts of Interest}

The authors declare that there are no conflicts of interest regarding the publication of this paper.

\section{Acknowledgments}

This research was supported by the Fundamental Research Funds for the Central Universities (grant no. HEUCFG201829), China Postdoctoral Science Foundation (2018M631911), and Heilongjiang Postdoctoral Fund (LBH-Z18055).

\section{References}

[1] J. Thornton, D. A. J. Pearce, D. Grace, M. Oodo, K. Katzis, and T. C. Tozer, "Effect of antenna beam pattern and layout on cellular performance in high altitude platform communications," Wireless Personal Communications, vol. 35, no. 1-2, pp. 35-51, 2005.

[2] Y. Wu, F. Hu, S. Kumar, J. D. Matyjas, Q. Sun, and Y. Zhu, "Apprenticeship learning based spectrum decision in multichannel wireless mesh networks with multi-beam antennas," IEEE Transactions on Mobile Computing, vol. 16, no. 2, pp. 314-325, 2017.

[3] F. R. Farrokhi, A. Lozano, G. J. Foschini, and R. A. Valenzuela, "Spectral efficiency of FDMA/TDMA wireless systems with transmit and receive antenna arrays," IEEE Transactions on Wireless Communications, vol. 1, no. 4, pp. 591-599, 2002.

[4] F. Jasbi and D. K. C. So, "Hybrid overlay/underlay cognitive radio network with MC-CDMA," IEEE Transactions on Vehicular Technology, vol. 65, no. 4, pp. 2038-2047, 2016.

[5] J. Lian and M. Brandt-Pearce, "Multiuser MIMO indoor visible light communication system using spatial multiplexing," Journal of Lightwave Technology, vol. 35, no. 23, pp. 5024-5033, 2017.

[6] I. R. Ishkaev, A. E. Shevelev, A. S. Ovsyannikova, S. V. Zavjalov, S. V. Volvenko, and S. B. Makarov, "Possibility of peak-to-avegare power ratio reduction by application of optimal signal for transmitter based on SDR HackRF one," in Proceedings of the IEEE International Conference on Electrical Engineering and Photonics (EExPolytech), St. Petersburg, Russia, October 2018.

[7] S. B. Makarov, A. S. Ovsyannikova, I. I. Lavrenyuk, S. V. Zavjalov, and S. V. Volvenko, "Distributions of probability of power values for random sequences of optimal FTN signals," in Proceedings of the International Symposium on Consumer Technologies (ISCT), St. Petersburg, Russia, May 2018.

[8] G. Bosco, A. Carena, V. Curri, P. Poggiolini, and F. Forghieri, "Performance limits of nyquist-WDM and CO-OFDM in high-speed PM-QPSK systems," IEEE Photonics Technology Letters, vol. 22, no. 15, pp. 1129-1131, 2010.

[9] A. Emmanuele, F. Zanier, G. Boccolini, and M. Luise, "Spread-spectrum continuous-phase-modulated signals for satellite navigation," IEEE Transactions on Aerospace and Electronic Systems, vol. 48, no. 4, pp. 3234-3249, 2012.

[10] G.-W. Lu, T. Sakamoto, A. Chiba et al., "Optical minimumshift-keying transmitter based on a monolithically integrated quad Mach-Zehnder in-phase and quadrature modulator," Optics Letters, vol. 34, no. 14, pp. 2144-2146, 2009.

[11] Z. Xie, G. Zhang, and D. M. Dongming Bian, "Constant envelope enhanced FQPSK and its performance analysis," Journal of Communications and Networks, vol. 13, no. 5, pp. 442-448, 2011.

[12] J. Vankka, M. Honkanen, and K. A. I. Halonen, "A multicarrier GMSK modulator," IEEE Journal on Selected Areas in Communications, vol. 19, no. 6, pp. 1070-1079, 2001.

[13] J. Li, Y. Peng, X. Jiang, Y. Yan, and P. Ranjan, "Enhanced index modulated OFDM spread spectrum," IEEE Access, vol. 6, pp. 71028-71037, 2018.

[14] H. Zhang, D. Le Ruyet, and M. Terre, "Spectral efficiency comparison between OFDM/OQAM- and OFDM-based CR networks," Wireless Communications and Mobile Computing, vol. 9, no. 11, pp. 1487-1501, 2009.

[15] X. Zhou, G. Y. Li, and G. Sun, "Low-complexity spectrum shaping for OFDM-based cognitive radio systems," IEEE Signal Processing Letters, vol. 19, no. 10, pp. 667-670, 2012.

[16] Q. Liu, Z. Zhou, S. H. Kan, and Y. B. Ye, "OFDM subcarriers coding for enhancing spectrum notch," in Proceedings of the International Symposium on Communications and Information Technologies, Lao, China, October 2008.

[17] Y. J. Zhang and K. B. Letaief, "Multiuser adaptive subcarrierand-bit allocation with adaptive cell selection for OFDM systems," IEEE Transactions on Wireless Communications, vol. 3, no. 5, pp. 1566-1575, 2004.

[18] N. Michailow, M. Matthé, I. S. Gaspar et al., "Generalized frequency division multiplexing for 5 th generation cellular networks," IEEE Transactions on Communications, vol. 62, no. 9, pp. 3045-3061, 2014.

[19] A. Farhang, N. Marchetti, and L. E. Doyle, "Low-complexity modem design for GFDM," IEEE Transactions on Signal Processing, vol. 64, no. 6, pp. 1507-1518, 2016.

[20] T. Ihalainen, A. Ikhlef, J. Louveaux, and M. Renfors, "Channel equalization for multi-antenna FBMC/OQAM receivers," IEEE Transactions on Vehicular Technology, vol. 60, no. 5, pp. 2070-2085, 2011. 
[21] T. Ihalainen, T. H. Stitz, M. Rinne, and M. Renfors, "Channel equalization in filter bank based multicarrier modulation for wireless communications," EURASIP Journal on Advances in Signal Processing, vol. 2007, no. 1, Article ID 49389, 2007.

[22] Y. D. Xu, W. Xue, and W. J. Shang, "A pan-function model for the utilization of bandwidth improvement and PAPR reduction," Mathematical Problems in Engineering, vol. 2014, Article ID 658093, 9 pages, 2014.

[23] M. Wen, B. Zheng, K. J. Kim et al., "A survey on spatial modulation in emerging wireless systems: research progresses and applications," IEEE Journal on Selected Areas in Communications, vol. 37, no. 9, pp. 1949-1972, 2019.

[24] J. Li, M. Wen, X. Cheng, Y. Yan, S. Song, and M. H. Lee, "Generalized precoding-aided quadrature spatial modulation," IEEE Transactions on Vehicular Technology, vol. 66, no. 2, pp. 1881-1886, 2017.

[25] W. Dmitry, "Increasing the speed of information transmission in radio communication systems through the use of spectraleffective signals," pp. 159-168 Omsk State Technical University of Russia, Omsk, Russia, 1999, Doctoral thesis. 


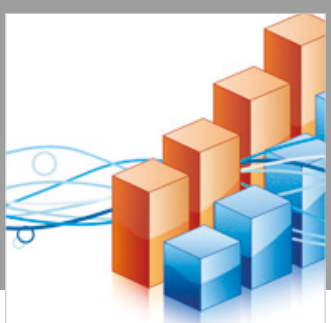

Advances in

Operations Research

\section{-n-m}
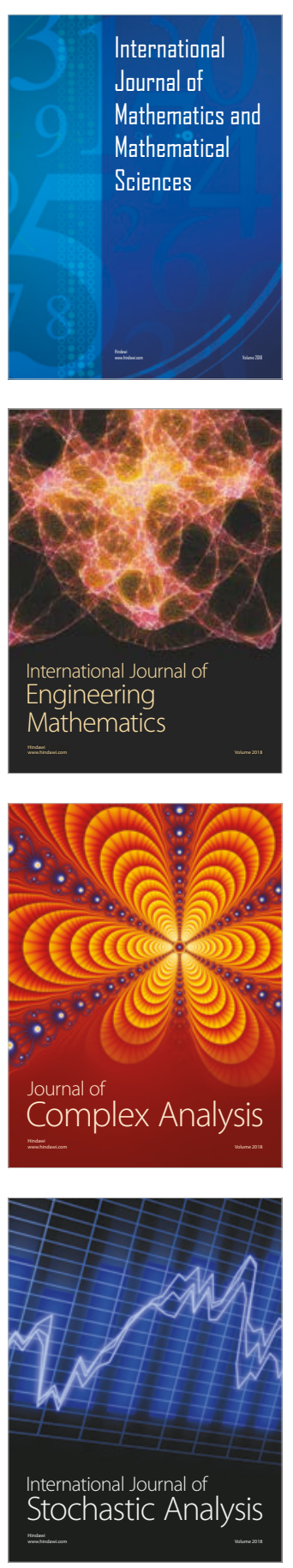
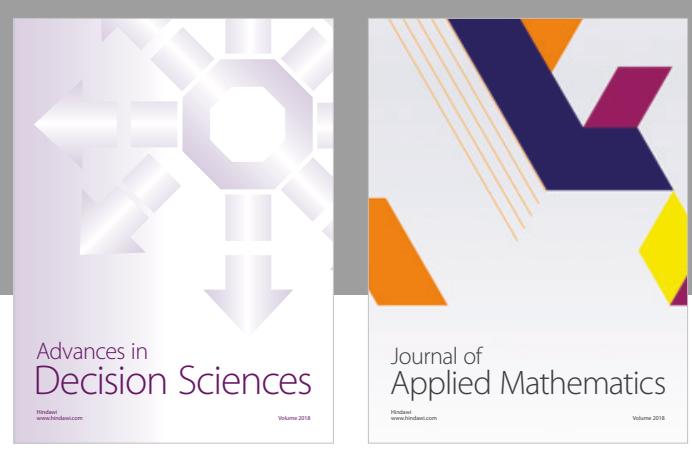

Journal of

Applied Mathematics
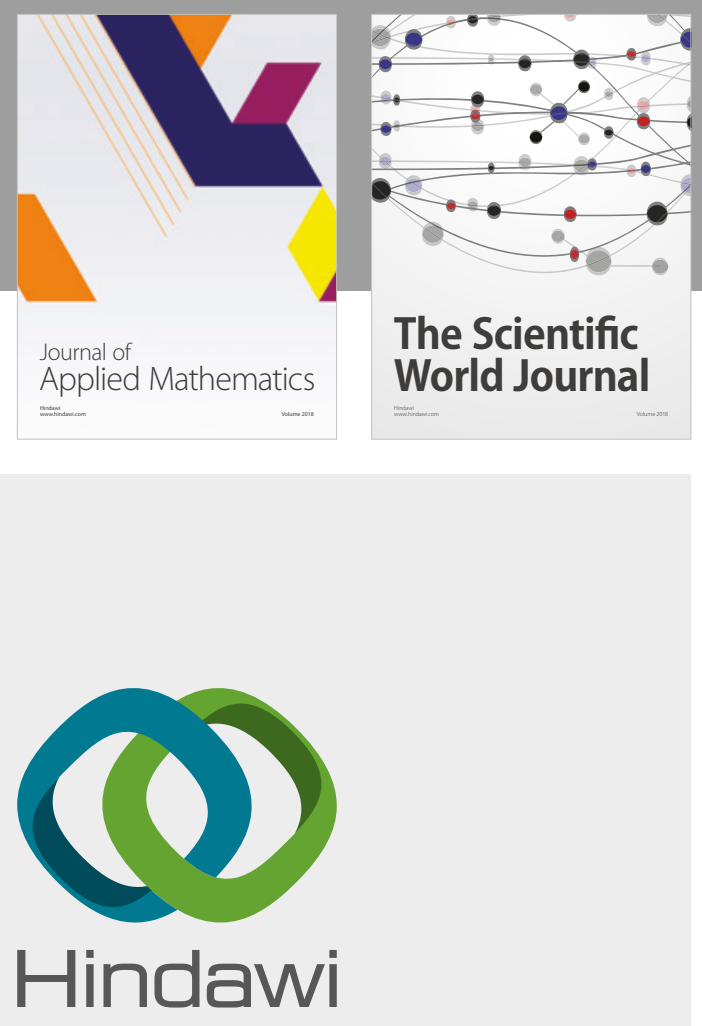

Submit your manuscripts at

www.hindawi.com

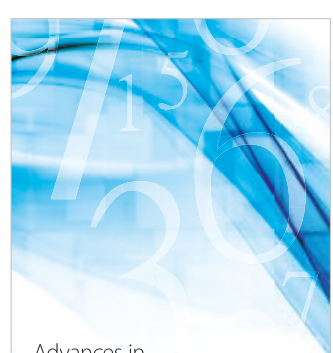

Advances in
Numerical Analysis
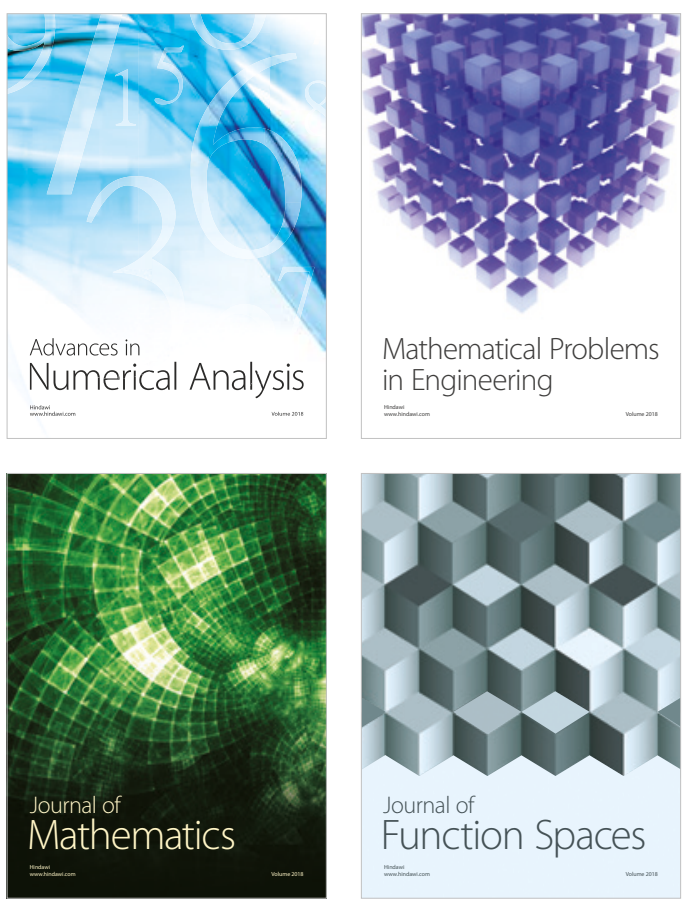

Mathematical Problems in Engineering

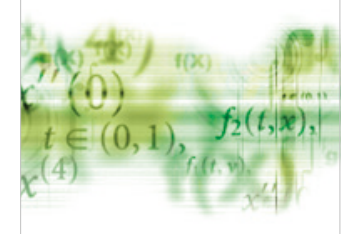

International Journal of

Differential Equations

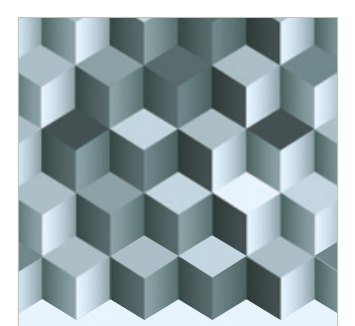

Journal of

Function Spaces

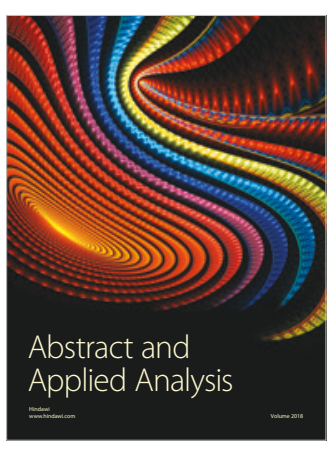

The Scientific

World Journal

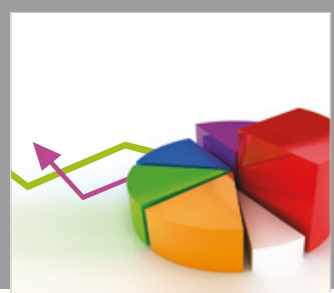

Journal of

Probability and Statistics
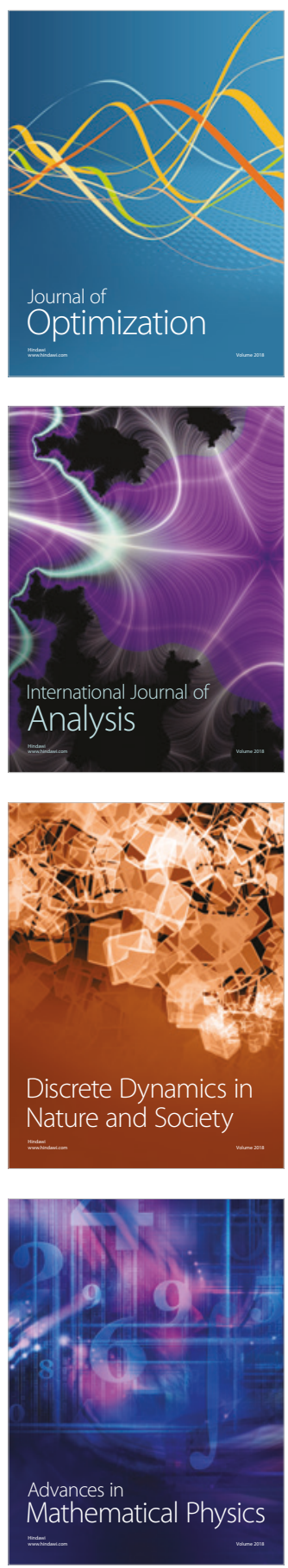